\title{
Tratamiento de la enfermedad pulmonar obstructiva crónica
}

\author{
F.J. FERNÁNDEZ-FERNÁNDEZ, E. AMENEIROS-LAGO', J. GONZÁLEZ \\ MORALEJA, G. PÍA IGLESIAS, F.S. MARTÍNEZ-DEBÉN, P. SESMA ${ }^{2}$ \\ Servicio de Medicina Interna y ${ }^{1}$ Unidad de Hospitalización a Domicilio. \\ Hospital Arquitecto Marcide. Ferrol. ${ }^{2}$ Departamento de Medicina. Universidad de \\ Santiago de Compostela. A Coruña.
}

TREATMENT OF CHRONIC OBSTRUCTIVE PULMONARY DISEASE

\begin{abstract}
RESUMEN
La enfermedad pulmonar obstructiva crónica (EPOC) es un proceso altamente prevalente y la mayoría de los estudios epidemiológicos han puesto de manifiesto que su prevalencia, morbilidad y mortalidad se han incrementado durante los últimos años. Investigaciones recientes sugieren una mejoría en el tratamiento. Así, varios estudios han demostrado la utilidad de los beta-2-agonistas inhalados de larga duración. Los glucocorticoides inhalados, aunque incapaces de alterar la progresión de la EPOC, pueden reducir la frecuencia de las exacerbaciones y mejorar la calidad de vida en un grupo limitado de pacientes. Asimismo, pueden ser beneficiosos en combinación con beta-agonistas de acción prolongada. Finalmente, un mejor conocimiento de los mecanismos moleculares y celulares implicados en la patogénesis de la EPOC debería conducir a tratamientos efectivos para lentificar o detener el curso de la enfermedad. Nuevas clases de estos fármacos, como los inhibidores de la fosfodiesterasa, están en el horizonte.
\end{abstract}

\begin{abstract}
Chronic obstructive pulmonary disease (COPD) is a highly preva lent condition and most epidemiological studies have found that COPD prevalence, morbidity and mortality have increased over the last few years. Recent trials suggest that treatments are improving. Several stu dies have demonstrated the usefulness of the long-acting inhaled beta-2agonists. Inhaled glucocorticoids, although unable to alter the progres sion of COPD, may reduce the frequency of acute exacerbations and health status deterioration in a limited group of patients with COPD. Likewise, these drugs may offer benefits in combination with long-acting inhaled beta-agonists. Finally, a better understanding of the molecular and cellular mechanisms involved in COPD pathogenesis should lead to effective treatments that slow or halt the course of the disease. New clas ses of agents such as the phosphodiesterase inhibitors are now in deve lopment.
\end{abstract}

KEY WORDS: Chronic obstructive pulmonary disease. Treatment.

PALABRAS CLAVE: Enfermedad pulmonar obstructiva crónica Tratamiento.

Fernández-Fernández FJ, Ameneiros-Lago E, González Moraleja J, Pía Iglesias G, Martínez-Debén FS, Sesma P. Tratamiento de la enfermedad pulmonar obstructiva crónica. An Med Interna (Madrid) 2003; 20: 148-155.

\section{INTRODUCCIÓN}

La enfermedad pulmonar obstructiva crónica (EPOC) se define como un síndrome caracterizado por el desarrollo de una limitación progresiva al flujo aéreo que, a diferencia del asma, no es completamente reversible $(1,2)$. El término EPOC abarca fundamentalmente dos entidades, la bronquitis obstructiva crónica y el enfisema. La bronquitis crónica, por contraste, se define por la presencia de tos productiva de más de 3 meses de duración durante más de 2 años consecutivos. La tos se debe a un aumento en la secreción de moco y no es necesario que se acompañe de limitación al flujo aéreo (1). Es evidente que el diagnóstico de la EPOC precisará de la realización de unas pruebas de función respiratoria (3).
Desde un punto de vista etiológico, en nuestro medio, el hábito de fumar es el principal agente implicado. La EPOC raramente ocurre en personas que no fuman y debe sospecharse la existencia de un déficit de alfa-1-antitripsina en dichas personas, así como en aquellos con antecedentes familiares y pacientes jóvenes (menores de 50 años) con enfisema. En esta revisión no se considerará el tratamiento de esta entidad específica. Otros factores a considerar en la etiología son la contaminación medioambiental, la exposición profesional a ciertos agentes químicos, gases o vapores y otros factores genéticoconstitucionales (1).

La EPOC es una entidad altamente prevalente. En los países de nuestro entorno supone la cuarta causa de muerte (4) y es la única causa común de muerte cuya incidencia está

Trabajo aceptado: 24 de noviembre de 2002

Correspondencia: Francisco José Fernández-Fernández. C/ Linares Rivas No 5-2º. Narón 15407. La Coruña. e-mail: fjf-fernandez@terra.es 
aumentando (1,5). En los últimos años se han producido discretos avances en la terapia y se han publicado diversos estudios que han llevado a un cambio en el algoritmo terapéutico del paciente estable con EPOC. En el futuro, un mejor conocimiento de los mecanismos celulares y moleculares implicados en el proceso inflamatorio permitirá el desarrollo de fármacos que puedan modificar la historia natural de esta enfermedad. En este artículo revisaremos el tratamiento actual del paciente estable con EPOC y haremos una breve mención de las perspectivas futuras del tratamiento.

\section{DIAGNÓSTICO DE LA EPOC}

Se sospechará la presencia de una EPOC en un paciente con disnea de esfuerzo de varios meses o años de duración. Podrá presentar o no síntomas acompañantes de bronquitis crónica. El diagnóstico requerirá la realización de unas pruebas de función respiratoria (3). Dichas pruebas, asimismo, podrán poner de manifiesto una limitación al flujo aéreo en individuos asintomáticos con factores de riesgo.

Excepto para la enfermedad confinada a la pequeña vía aérea, la clave del diagnóstico es la presencia de un patrón obstructivo, con una disminución en el cociente $\mathrm{FEV}_{1} / \mathrm{FVC}$. El $\mathrm{FEV}_{1}$ está disminuido, generalmente, con una reducción asociada de la FVC (capacidad vital forzada). Como regla, la VC con una espiración lenta también es baja. Una mayor reducción de la FVC que la VC con una espiración lenta indica atrapamiento aéreo. Frecuentemente, los volúmenes pulmonares son anormalmente altos. Típicamente, la capacidad funcional residual, la capacidad pulmonar total y el volumen residual están aumentados.

Aunque el enfisema y la bronquitis crónica normalmente coexisten, ocasionalmente uno de ellas predomina de forma marcada. En este supuesto la determinación de la capacidad de difusión de $\mathrm{CO}\left(\mathrm{DL}_{\mathrm{CO}}\right)$ será útil. En el enfisema habrá una disminución de la $\mathrm{DL}_{\mathrm{CO}}$, mientras que en la bronquitis crónica será normal o casi normal.

En la enfermedad de la pequeña vía aérea los flujos espiratorios son usualmente normales, excepto a volúmenes pulmonares bajos. El $\mathrm{FEF}_{25-75 \%}$ y el $\mathrm{FEF}_{75}$ estarán reducidos, aunque este hallazgo no es totalmente específico. $\mathrm{La} \mathrm{DL}_{\mathrm{Co}}$ y los volúmenes pulmonares serán normales.

La realización de las pruebas de función respiratoria nos permitirá establecer un diagnóstico y cuantificar la severidad de la enfermedad, lo cual, como posteriormente veremos, tendrá implicaciones importantes en el tratamiento. Asimismo, podremos valorar la respuesta broncodilatadora y nos proporcionará valiosa información sobre los cambios de la función pulmonar con el tiempo.

\section{TRATAMIENTO DEL PACIENTE ESTABLE CON EPOC}

En el tratamiento de la EPOC, desde un punto de vista práctico, podemos considerar aquellas medidas que han demostrado mejorar la supervivencia (interrupción del tabaquismo y oxigenoterapia en el paciente con hipoxemia crónica severa) y aquellas otras que mejoran los síntomas y la calidad de vida (6).

Se considera que el paciente está estable si no tuvo agudizaciones, la $\mathrm{PaO}_{2}$ y la $\mathrm{PaCO}_{2}$ no tuvieron modificaciones superiores a los $5 \mathrm{~mm} \mathrm{Hg}$ y las variaciones del FEV1 fueron inferiores al $10 \%$ en los 3 últimos meses. Proponemos el algoritmo de la figura 1.

1. Abandono del hábito de fumar. El avance en la limitación del flujo aéreo se puede frenar con el reconocimiento precoz de la alteración de la función pulmonar y la eliminación de los factores de riesgo. El tabaquismo es el principal factor de riesgo ambiental para la aparición de la EPOC, aunque ésta afecta a sólo un porcentaje de fumadores, lo que indica que otros factores de riesgo son también importantes. Como el Lung Health Study (7) demostró, la interrupción del tabaquismo reducirá el descenso acelerado del $\mathrm{FEV}_{1}$ que se observa en algunos fumadores. Así pues, se informará adecuadamente al paciente sobre la importancia de eliminar esta adicción y se le proporcionará tratamiento farmacológico, si fuese preciso, para el síndrome de abstinencia. A este respecto, disponemos de la terapia de sustitución nicotínica y de otros fármacos, entre los que destaca el antidepresivo noradrenérgico bupropion $(8,9)$.

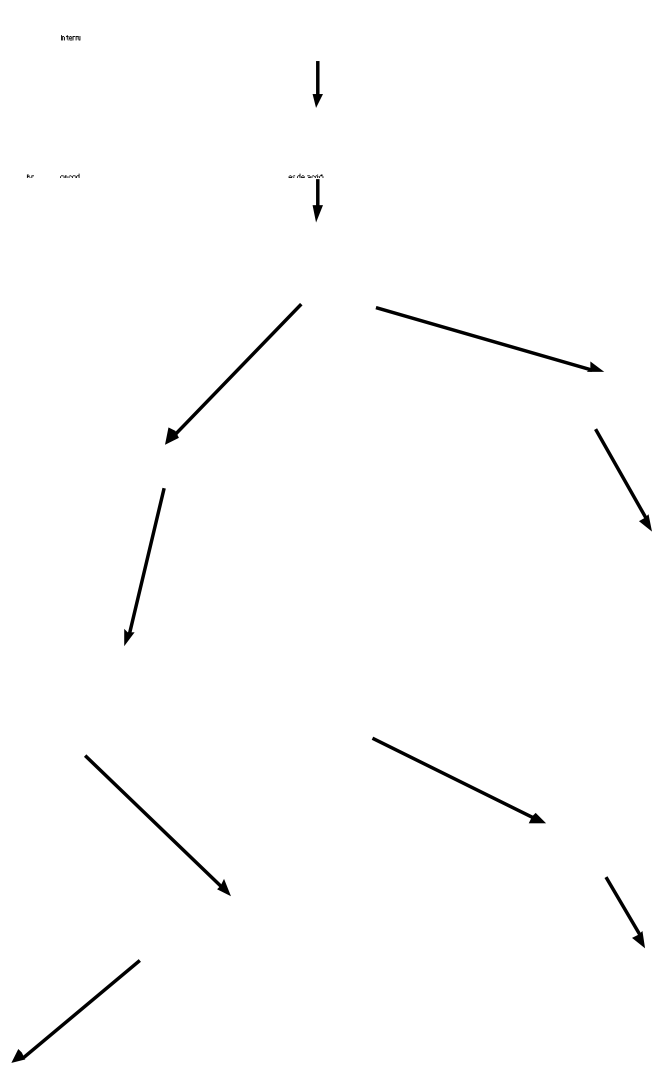

Fig. 1. Algoritmo terapéutico en el paciente con EPO C estable. 
2. Tratamiento broncodilatador. Estos fármacos producen aumentos muy discretos del $\mathrm{FEV}_{1}(<10 \%)$ y no disponemos de pruebas firmes de que el uso regular de los mismos pueda alterar el avance de la EPOC. Sin embargo, alivian los síntomas y mejoran la tolerancia al ejercicio, por lo que resultan útiles en pacientes sintomáticos, a pesar de la escasa mejoría que se objetiva en las pruebas espirométricas (10). En pacientes con síntomas esporádicos se podrían usar broncodilatadores de acción corta a demanda y en aquellos con síntomas persistentes se aconseja el tratamiento con anticolinérgicos o betaagonistas de larga duración, sobre una base regular (3).

-Anticolinérgicos: un mejor conocimiento de las vías colinérgicas pulmonares y el desarrollo de agentes anticolinérgicos más selectivos que aquellos de los que se disponía inicialmente (derivados de la belladona) llevó a que estos fármacos se considerasen por algunos autores el tratamiento de elección para pacientes con EPOC estable sintomática (11). Los agentes anticolinérgicos actuales (ipratropio y tiotropio, éste último en investigación) no atraviesan fácilmente las barreras biológicas, lo que minimiza los efectos secundarios. Estos fármacos tienen un inicio de acción más lento que los $\beta$-agonistas de acción corta y proporcionan una broncodilatación más duradera. Asimismo, en adición a la broncodilatación, disminuyen la hipersecreción de moco, mejoran la tolerancia al ejercicio y la calidad del sueño. Los anticolinérgicos pueden también producir broncodilatación en algunos pacientes con EPOC que no responden a los $\beta$-agonistas de corta duración y su uso proporciona efectos duraderos sin taquifilaxia, a diferencia del uso de los $\beta$-agonistas de acción corta a largo plazo. Con el ipratropio, la dosis habitual de 2 inhalaciones cada 6 horas, puede ser doblada o triplicada sin notables efectos secundarios. Si este fármaco puede mejorar o no la función pulmonar a largo plazo es objeto de controversia, aunque parece que su empleo no modifica la progresión de la EPOC. Así, en el Lung Health Study (7), pacientes fumadores con edades comprendidas entre 35 y 60 años y EPOC leve eran distribuidos en 3 grupos: interrupción del tabaquismo, interrupción del tabaquismo más ipratropio y no intervención. El tratamiento con ipratropio producía un aumento en el $\mathrm{FEV}_{1}$ que era evidente al final del primer año, sin embargo, este efecto desaparecía al discontinuar el fármaco y el uso del broncodilatador no tenía influencia en el deterioro de la función pulmonar a largo plazo. En contraste, en un estudio comparativo de 7 ensayos clínicos (12) en los que el ipratropio era comparado con un $\beta$-agonista, administrados durante 90 días, los autores concluían que el ipratropio mejoraba la función pulmonar basal y podría mejorar la respuesta a la broncodilatación aguda, efectos que no eran observados con los $\beta$-agonistas. En este último estudio los pacientes tenían una disfunción pulmonar más severa y se incluían pacientes fumadores y no fumadores, con un mayor beneficio en estos últimos. Una diferencia importante entre ambos estudios era la duración del tiempo de retirada del broncodilatador (12 horas frente a 40 horas) antes del estudio espirométrico y quizás un efecto broncodilatador residual a las 12 horas podría explicar las diferencias (13).

- $\beta$-agonistas: se pueden utilizar como tratamiento inicial en monoterapia o asociados a los anticolinérgicos. La adición de un $\beta$-agonista, a dosis standard, a un anticolinérgico produce resultados superiores a los que se obtienen con ambos fármacos de forma aislada (14), mientras se minimizan los efectos secundarios asociados al uso de altas dosis de los agentes de forma individual. En el paciente estable puede resultar más conveniente la utilización de $\beta$-agonistas de acción prolongada, como el salmeterol y el formoterol (15-17). Ello permite su administración 2 veces al día, una mejor cumplimentación terapéutica y un mejor control de los síntomas respiratorios, sobre todo nocturnos. Un beneficio adicional con estos fármacos podría ser una disminución de las exacerbaciones de origen infeccioso, ya que reducen la adhesión de bacterias como el Haemophilus influenzae a las células epiteliales del tracto respiratorio (18). Aunque la estimulación $\beta$ puede mejorar la función ciliar, también estimula la producción de moco por las glándulas submucosas sin estimular la secreción serosa, lo que lleva a un aumento en el espesor del moco (19). Otro efecto deletéreo a tener en cuenta es la posibilidad de hipokaliemia con dosis elevadas.

Los $\beta$-agonistas pueden utilizarse por vía oral, subcutánea, intravenosa o inhalada, siendo esta última la de elección para minimizar los efectos secundarios. No obstante, en pacientes con EPOC estable que no colaboran (edad avanzada, demencia, etc.) podríamos recurrir a la vía oral.

-Teofilinas: en pacientes con EPOC estable las teofilinas son agentes de $3^{\text {a }}$ línea (2) ya que su acción broncodilatadora es limitada y sus límites terapéuticos estrechos. No obstante, su administración en preparados de larga acción por la tardenoche puede reducir el declinar nocturno del $\mathrm{FEV}_{1}$ y mejorar los síntomas respiratorios matutinos. Las teofilinas pueden también mejorar la función de los músculos respiratorios, el aclaramiento mucociliar, producir vasodilatación pulmonar e incluso reducir la inflamación de la vía aérea (20). Por tanto, se podrían utilizar entre los pacientes que no han respondido de forma adecuada a los fármacos de primera línea y también son útiles en pacientes que no utilizan de forma adecuada la vía inhalada. Se aconseja que los niveles séricos de la teofilina se mantengan entre 8-15 mg/l (11). Se tendrá en cuenta la dosis en función de la situación clínica del paciente y las frecuentes interacciones con otros medicamentos. Entre sus efectos secundarios destacan el temblor y los síntomas gastrointestinales, en particular el reflujo gastroesofágico. Complicaciones de más riesgo son las arritmias y convulsiones (21), que pueden ocurrir en ancianos a niveles tan bajos como $14 \mathrm{mg} / \mathrm{l}$.

3. Corticosteroides. En los esquemas terapéuticos clásicos (2) los corticoides orales eran añadidos al tratamiento de los pacientes que no se controlaban adecuadamente con anticolinérgicos, $\beta$-agonistas y teofilinas. Si se documentaba mejoría, éstos se disminuían a la dosis mínima eficaz (0-10 mg/día o a días alternos). En los últimos años se han publicado una serie de estudios con diferentes glucocorticoides inhalados (22-25) con el objetivo de evaluar su utilidad en lentificar el deterioro de la función pulmonar que ocurre en los pacientes con EPOC. Tomados en conjunto podemos decir que los corticoides inhalados no se han mostrado útiles para frenar el descenso del $\mathrm{FEV}_{1}$. Sin embargo, en pacientes con EPOC moderadasevera disminuyen los síntomas respiratorios y la frecuencia de las exacerbaciones. También se ha objetivado una disminución en la reactividad de la vía aérea a la metacolina y su potencial utilidad en pacientes con eosinofilia en el esputo. Sobre la base de los ensayos clínicos publicados hasta la fecha podemos concluir que los glucocorticoides inhalados pueden ser recomendados en pacientes sintomáticos con EPOC moderada-severa o para aquellos con exacerbaciones frecuentes, pero no para pacientes con EPOC leve (26). Las guías actuales aconsejan un ensayo de 6 semanas a 3 meses con glucocor- 
ticoides inhalados para identificar a aquellos pacientes con EPOC que se puedan beneficiar del tratamiento esteroideo a largo plazo. Un aspecto importante a tener en cuenta es el hecho de que un ciclo corto de glucocorticoides orales es un mal predictor de una potencial respuesta a largo plazo a los esteroides inhalados.

Se complementará el esquema terapéutico propuesto en la figura 1 con las siguientes medidas adicionales:

1. Oxigenoterapia crónica domiciliaria

2. Educación en el uso de la vía inhalatoria

3. Rehabilitación pulmonar y nutrición.

4. Manejo del insomnio, depresión y ansiedad.

5. Vacunación.

1. Oxigenoterapia crónica domiciliaria (OCD). La administración continua de oxígeno en el hogar mejora la supervivencia de los pacientes con EPOC e hipoxemia crónica severa $\left(\mathrm{PaO}_{2}<55 \mathrm{mmHg}\right)(27,28)$. Otros efectos beneficiosos consisten en reducción de la policitemia, de la presión arterial pulmonar y de la tolerancia al ejercicio. El oxígeno puede también mejorar los resultados de las pruebas neuropsiquiátricas y reducir las arritmias nocturnas. Antes de indicar la oxigenoterapia continua a un paciente con EPOC se ha de constatar la presencia de hipoxemia y que se trata de una situación crónica. El paciente debe estar en una situación estable, sin signos de infección broncopulmonar ni insuficiencia cardíaca congestiva, así como ausencia de variaciones significativas en el $\mathrm{FEV}_{1}, \mathrm{PaO}_{2}, \mathrm{PaCO}_{2}$ y pH, en al menos 2 controles separados 3 semanas aparte. Este aspecto es especialmente importante ya que algunos pacientes en insuficiencia respiratoria desencadenada por una reagudización pueden no requerir oxigenoterapia crónica una vez superada esta fase. También es importante asegurarse de que el paciente en situación estable recibe y realiza el tratamiento correcto (29). Hay que señalar que la mayoría de los autores aconsejan no dar una indicación definitiva para la OCD hasta que pasen 3 meses, ya que un 20-40\% de los pacientes tras este tiempo no cumplirán los criterios para la OCD.

Las indicaciones de la OCD se muestran en la tabla I. La dosis de oxígeno debe ser suficiente para mantener una $\mathrm{PaO}_{2}$ $>60 \mathrm{mmHg}$ o una $\mathrm{SaO}_{2}>90 \%$ a nivel del mar. El tiempo total diario debe ser el máximo posible y nunca inferior a 15 horas al día, siendo recomendable evitar períodos superiores a 2 horas consecutivas sin administración de oxígeno.

En lo que a la OCD se refiere existen varias situaciones concretas dignas de mencionar. Existe un cierto número de pacientes con EPOC e hipoxemia durante el sueño y valores normales o casi normales durante la vigilia. Estudios con oxigenoterapia nocturna aislada no mostraron mejoría en la supervivencia ni retraso en la prescripción de OCD a largo plazo (30). Por otra parte, pacientes que no precisan OCD

\section{TABLA I}

INDICACIO NES PARA OXIGENO TERAPIA CRÓ NICA DOM ICILIARIA EN LA EPOC

$\mathrm{PaO}_{2} \leq 55 \mathrm{~mm} \mathrm{Hg} O \mathrm{SaO}_{2} \leq 89 \%$

$\mathrm{PaO}_{2}$ de 56 a $59 \mathrm{~mm} \mathrm{Hg} 0 \mathrm{SaO}_{2} \leq 90 \%$, si: hipertensión pulmonar, cor pulmonale, policitemia o función neuropsíquica alterada por la hipoxemia pueden requerir oxigenoterapia suplementaria durante los viajes en avión. A 8.000 pies de altitud la $\mathrm{PaO}_{2}$ puede disminuir $25 \mathrm{mmHg}$ por debajo de la existente a nivel del mar. De ahí que, en pacientes con valores en torno a $70 \mathrm{mmHg}$ a nivel del mar, puede ser necesario la administración de oxígeno en vuelos prolongados (31).

La mejoría en la oxigenación mediante el tratamiento adecuado, que puede requerir de la $\mathrm{OCD}$, es la forma más fisiológica de corregir la policitemia que presentan algunos pacientes. Dicha eritrocitosis aumenta la viscosidad de la sangre y agrava la hipertensión pulmonar. Puede, asimismo, desencadenar una sensación de plenitud en la cabeza y cefaleas. Estos pacientes pueden tener una respuesta subjetiva favorable a la flebotomía periódica cuando el hematocrito es mayor del 55\%. En apoyo de esta mejoría subjetiva está la observación de que, tras la flebotomía, el flujo cerebral previamente reducido vuelve a la normalidad (31).

2. Educación en el uso de la vía inhalatoria. No existe ninguna duda de que la vía inhalatoria es la de elección para administrar fármacos broncodilatadores en pacientes con EPOC. Tan importante como prescribir un tratamiento correcto es asegurarse de que el paciente entiende y cumple nuestras recomendaciones. Desafortunadamente, muchos profesionales de la salud son también incapaces de usar o demostrar como se deben utilizar de forma correcta los sistemas de inhalación $(32,33)$. Los factores que determinan el depósito de fármacos en la vía aérea dependen del generador de aerosol y de las características del paciente $(34,35)$. Una buena parte de las partículas generadas por el aerosol chocan contra la superficie de la vía aérea y se produce el depósito por un mecanismo de "impactación". Otras partículas se depositan por sedimentación gravitacional. Las partículas $<2 \mu$ de diámetro son depositadas en su mayor parte en los alvéolos, con un depósito máximo para aquellas de $3 \mu$. Partículas en el rango de 5 a $10 \mu$ de diámetro se depositan principalmente en la orofaringe y las vías de conducción aérea de mayor tamaño. Así, las partículas $<5 \mu$ pueden considerarse como pertenecientes al "rango respirable" de los aerosoles terapéuticos, aunque incluso, dentro de este rango, algunas partículas se depositarán en la orofaringe y otras serán exhaladas. Por parte del paciente, su flujo inspiratorio influye en el tipo y cantidad de partículas depositadas. Un flujo alto (>100 1/min) hace predominar el depósito por impactación, pero consigue una elevada penetración de partículas. Un flujo inspiratorio bajo (<30 1/min) aunque favorece la sedimentación, hace peligrar la cantidad de sustancia inhalada. El flujo ideal oscila entre 30 y 60 1/min. El volumen inspiratorio también condiciona la cantidad de fármaco inhalado. Un volumen bajo dificulta la correcta penetración y, por contra, un volumen alto favorece la entrada de una buena cantidad del fármaco. Otro aspecto importante es la realización de un tiempo de apnea postinspiratoria para favorecer el depósito por el mecanismo de sedimentación. En este apartado describiremos de forma breve los principales sistemas que tenemos para la administración de fármacos por esta vía.

- Cartucho presurizado: los sistemas de inhalación que utilizan este sistema se denominan inhaladores presurizados de dosis controlada (MDI, metered-dose inhalers, en la literatura anglosajona). Producen partículas de 2 a 4 micras y están constituidos por 3 elementos. Un cartucho o dispositivo cilíndrico metálico, que contiene el medicamento activo en solución o suspensión en un gas propelente y a una presión de 3-4 
atmósferas. La válvula dosificadora, que permite liberar una dosis controlada del fármaco micronizado con cada pulsación y el envase externo de plástico, en el que se encaja el cartucho. Los aerosoles presurizados son de pequeño tamaño, fáciles de transportar, no necesitan energía externa para su funcionamiento y se pueden adaptar a circuitos de ventilación asistida. Asimismo, la limpieza y mantenimiento son muy sencillos. Su principal inconveniente es la dificultad de coordinación entre la inspiración y el disparo (35). Por otra parte, la impactación del fármaco en la orofaringe puede tener un efecto irritante e interrumpir la maniobra de inhalación (efecto frío del freón). Otro inconveniente es el efecto negativo de los propelentes fluorocarbonados sobre la capa de ozono atmosférica.

-Cámaras de inhalación: son aparatos diseñados para mejorar la eficiencia en el uso de los cartuchos presurizados $(36,37)$. Estos dispositivos, al aumentar la distancia entre el cartucho y la boca, enlentecen el flujo del aerosol y reducen la impactación en la orofaringe (en teoría, menores efectos irritantes y disminuyen la aparición de candidiasis oral tras la administración de corticoides) (36,38). Asimismo, la evaporación de los fluorocarbonos en su interior disminuye el tamaño de las partículas y favorece su depósito en las vías pulmonares periféricas. Una de sus principales ventajas es que no se precisa la sincronización entre la inspiración y la administración del aerosol. Su principal desventaja es el tamaño, que hace incómodo su transporte.

-Dispositivos de polvo seco: los inhaladores de polvo seco generan partículas de 1-2 micras de diámetro y el flujo inspiratorio ideal para una correcta inhalación del fármaco oscila entre 30 y $60 \mathrm{l} / \mathrm{min}$. Dentro de los inhaladores de polvo multidosis disponemos, fundamentalmente, de los sistemas Turbuhaler $^{\circledast}$ y Accuhaler ${ }^{\circledast}$. El sistema Turbuhaler ${ }^{\circledast}$ proporciona 200 dosis del fármaco micronizado y no contiene aditivos. El tamaño de las partículas es de alrededor de 1 micra y una ventana muestra una señal roja cuando sólo quedan 20 dosis de fármaco. En el sistema Accuhaler ${ }^{\circledR}$ el fármaco viene dispuesto en una tira autoenrrollable en forma de blíster u óvulo. Cada vez que se acciona el gatillo del dispositivo, un óvulo es desplazado hacia la zona de inhalación y es simultáneamente agujereado. Tiene un indicador en el que muestra el número de dosis que restan.

La eficacia de los dispositivos de polvo seco es igual o superior a la obtenida por los cartuchos presurizados $(39,40)$. La facilidad de su empleo hace que desaparezca el problema de coordinación pulsación-inspiración y no utilizan gases propelentes contaminantes. Entre sus inconvenientes destacan que precisan una inspiración voluntaria, con un flujo inspiratorio ideal entre 30 y 60 l/min. El impacto orofaríngeo del fármaco es elevado con lo que aumentan los efectos secundarios locales y el precio es superior al de los cartuchos presurizados.

-Nebulizadores: el fármaco a nebulizar se añade a un disolvente (suero salino o agua bidestilada estéril) y el nebulizador generará partículas de aerosol de diferentes tamaños al romper la tensión superficial del líquido y crear una fina niebla. Estas partículas pueden ser acuosas y utilizarse como un elemento hidratador. El tamaño de las partículas determina la migración de las mismas. Aquellas entre 4 y 10 micras quedan atrapadas en la nasofaringe, mientras que las inferiores a 4 micras sobrepasan las vías aéreas superiores y se depositan en los bronquiolos terminales. Los nebulizadores de chorro o jet se basan en los principios de Bernoulli y de Venturi. En los ultrasónicos, las gotas de aerosol se producen por ondas de sonido de alta frecuencia generadas por un cristal piezoeléctrico (35). Estos sistemas precisan de una menor colaboración del paciente y son ampliamente utilizados en los Servicios de Urgencia hospitalarios para el tratamiento de las reagudizaciones de la EPOC (41). Entre sus inconvenientes destacan su coste elevado, gran tamaño, retención del fármaco en las paredes de los equipos, aumento de las posibilidades de contaminación bacteriana y necesidad de una fuente de energía para su empleo.

3. Rehabilitación pulmonar y nutrición. Los programas de ejercicio, aunque no mejoran la función pulmonar, se acompañan de un aumento en la tolerancia al mismo y de sensación de bienestar. Los mecanismos responsables para esta mejoría no están claros. Se ha sugerido que, al progresar con los programas de entrenamiento, los pacientes se desensibilizarían a la disnea inducida por la carga ventilatoria (42). Otros autores han postulado una reducción en la acidosis láctica con el ejercicio (43). Cualquier paciente capaz de someterse a rehabilitación se beneficiará de un programa que incluya ejercicios con las piernas. Los ejercicios de los miembros superiores se toleran mal, ya que algunos de dichos músculos tienen una doble función, postural y respiratoria. El ejercicio con las extremidades superiores produce mejoría de las actividades específicas que se efectúan con los brazos y, dada su sencillez, deberían incluirse en la rehabilitación de los pacientes que se quejan de disnea con la actividad de los brazos (6).

Si existe malnutrición, los suplementos dietéticos pueden mejorar la fuerza muscular, disminuir la fatigabilidad y mejorar la disnea. Antes de comenzar con el suplemento dietético es necesario realizar una detallada historia clínica para descartar causas graves de bajo peso y pérdida ponderal.

4. Manejo del insomnio, depresión y ansiedad. Pacientes con EPOC pueden tener importantes problemas de ansiedad y depresión relacionados con el estrés y la incapacidad asociada a su enfermedad. Por otra parte, no es infrecuente el infratratamiento por el temor a producir depresión respiratoria con la medicación. La depresión no es detectada con frecuencia (44) y se puede manifestar como ansiedad o insomnio. Dentro de los diferentes fármacos, los inhibidores selectivos de la recaptación de la serotonina (44) (ISRS) son particularmente útiles en el tratamiento de la depresión y de los ataques de pánico. Otros 2 antidepresivos útiles son la nefazodona, con ligeras propiedades sedantes (útil en pacientes con insomnio) y el bupropion, que también se puede prescribir en pacientes que necesitan apoyo farmacológico para su abstinencia tabáquica (9).

La ansiedad es común en pacientes con EPOC (45), con ataques de pánico en un $25 \%$ de los pacientes. La ansiedad debe diferenciarse de la disnea, ya que el tratamiento broncodilatador adecuado puede evitar el empleo de ansiolíticos. Por otra parte, la ansiedad puede desencadenar sintomatología disneica que no responda a los broncodilatadores. Los ISRS pueden usarse para tratar la ansiedad con relativa seguridad. La buspirona es un ansiolítico que no produce depresión del centro respiratorio $(11,45)$, aunque sus efectos son modestos y pueden no ser suficientes. Si se precisa un tratamiento prolongado para la ansiedad, la combinación de un ISRS y una benzodiacepina a dosis bajas puede ser útil.

Los trastornos del sueño también son frecuentes. Antes de iniciar tratamiento inductor del sueño se realizará una anam- 
nesis adecuada con polisomnografía (46) u oximetría nocturna si fuese preciso. Si es necesario prescribir un fármaco hipnótico, los antihistamínicos con acción sedante pueden constituir una buena opción inicial con bajo riesgo.

5. Vacunación. La vacunación antineumocócica y frente al virus de la gripe son importantes medidas preventivas en pacientes con EPOC aconsejadas por el Advisory Committee on Immunization Practices $(47,48)$. La vacuna antineumocócica actual cubre más del $80 \%$ de cepas neumocócicas y es efectiva en más del $60 \%$ de sujetos inmunocompetentes para producir anticuerpos después de una sola dosis.

La vacunación antigripal se ha mostrado efectiva en reducir las tasas de hospitalización por gripe o neumonía y reduce la tasa de mortalidad (49). Actualmente también disponemos de los inhibidores de la neuraminidasa que pueden ser útiles, en algunos pacientes, para prevenir el desarrollo de la gripe, o bien, reducir los síntomas cuando se toman de forma precoz en el curso de la enfermedad $(50,51)$.

\section{CIRUGÍA DE REDUCCIÓN DE VOLUMEN PULMONAR}

Las importantes limitaciones del tratamiento médico para producir mejoría sintomática en los pacientes con enfisema han llevado a la búsqueda de distintas soluciones quirúrgicas. En 1957 Brantigan y Mueller describieron una técnica que consistía en la resección quirúrgica de tejido pulmonar para reducir el volumen del parénquima pulmonar hiperinsuflado, denominándose a ésto, cirugía de reducción del volumen pulmonar. Aunque el 75\% de los pacientes referían mejoría clínica, no había una documentación objetiva de dicho beneficio y el procedimiento se acompañaba de una alta mortalidad operatoria (18\%). Más recientemente, en 1995 Cooper y cols. describieron una modificación de la técnica anterior en la cual realizaban una resección de tejido pulmonar bilateral vía esternotomía media. Se han intentado también la cirugía toracoscópica video-asistida y la ablacción con láser. Todas estas aproximaciones se basan en que la reducción en la hiperinsuflación, mediante la retirada de las partes más enfisematosas del pulmón, mejora la función ventilatoria. Se han publicado los resultados de numerosos estudios de cirugía de reducción de volumen pulmonar con resultados contradictorios $(52,53)$. Aunque ciertas características, como son la presencia de enfisema localizado en los lóbulos superiores o unas resistencias pulmonares bajas durante la inspiración parecen predecir mejores resultados, lo cierto es que esta técnica plantea aún numerosas incógnitas, como son: definir el beneficio a largo plazo de este tipo de cirugía, que procedimiento es el más idóneo o bien qué subgrupo de pacientes con enfisema serían los más beneficiados. Para tratar de dar respuesta a estas preguntas se puso en marcha un estudio aleatorizado y multicéntrico, The National Emphysema Treatment Trial) (54). Los resultados preliminares de este estudio muestran que entre los pacientes con una disminución severa de la función pulmonar, la cirugía ocasiona más muertes que el tratamiento médico durante los primeros 30 días y, asimismo, la situación clínica de aquellos que sobreviven a la cirugía no es sustancialmente mejorada. Este estudio ha definido un subgrupo de pacientes en los que la cirugía de reducción de volumen no constituye una alternativa terapéutica. En dicho subgrupo se incluyen aquellos con un $\mathrm{FEV}_{1}$ del $20 \%$ o menor del valor de referencia, una capacidad de difusión del monóxido de carbono del
$20 \%$ o menor del valor teórico y aquellos con una distribución homogénea del enfisema en la tomografía computarizada.

\section{PERSPECTIVAS FUTURAS DE TRATAMIENTO EN LA EPOC}

En contraste con el desarrollo en el conocimiento y tratamiento del asma, la EPOC ha recibido menor atención y se han introducido pocos fármacos nuevos para esta importante proceso $(55,56)$. En la EPOC existe un proceso inflamatorio activo y una lesión proteolítica progresiva del parénquima pulmonar. Existen actualmente varias clases de fármacos en desarrollo clínico y preclínico que son presentados en la tabla II.

1. Antagonistas de los mediadores. En la EPOC están implicados varios mediadores inflamatorios y hay un proceso inflamatorio continuo, incluso en los pacientes que han dejado de fumar. La EPOC se caracteriza por una inflamación neutrofílica y, por tanto, la atención se ha centrado en aquellos mediadores implicados en el reclutamiento y la activación de los neutrófilos, así como en los que derivan del estrés oxidativo, que también está aumentado en la EPOC.

Entre los antagonistas de los mediadores destacan los inhibidores del leucotrieno B4 (LTB4). El LTB4 es un potente factor quimiotáctico para los neutrófilos que se encuentra aumentado en el esputo de pacientes con EPOC. Probablemente deriva de los macrófagos alveolares y de los propios neutrófilos y su acción parece sinérgica con la de la interleucina 8 (IL8), también implicada en el reclutamiento de los neutrófilos. Actualmente están en desarrollo varios antagonistas selectivos de los receptores de leucotrienos. Los inhibidores de la 5- lipooxigenasa (5-LO), que previenen la síntesis del leucotrieno B4, también están en estudio en la EPOC. Como decíamos previamente, la IL8 está implicada en la quimiotaxis de los neutrófilos y se encuentra aumentada en el esputo de los pacientes con EPOC. Por esta razón, se está investigan-

\section{TABLA II}

\section{POTENCIALES TRATAM IENTOS PARA LA EPO C}

Antagonistas de los mediadores:
Antagonistas del leucotrieno B4
Inhibidores de la 5 'lipooxigenasa (5-LO)
Antagonistas de la interleukina 8 (IL-8)
Inhibidores de TNF $\alpha$
Antioxidantes
Fármacos antiinflamatorios:
Inhibidores de la fosfodiesterasa 4
Inhibidores del factor nuclear $\kappa B$
Inhibidores de las moléculas de adhesión
Interleukina 10 (IL-10)
Inhibidores de proteasas:
Inhibidores de la elastasa de neutrófilos
Inhibidores de la catepsina
Inhibidores de las metaloproteinasas de matriz
$\alpha 1$ antitripsina
Elafina
Inhibidor de la leucoproteasa secretora

Antagonistas del leucotrieno B4

Inhibidores de la 5 'lipooxigenasa (5-LO)

Antagonistas de la interleukina 8 (IL-8)

Inhibidores de TNF $\alpha$

ármacos antiinflamatorios:

Inhibidores de la fosfodiesterasa 4

nhibidores del factor nuclear $\mathrm{kB}$

Inhibidores de las moléculas de adhesión

Interleukina 10 (IL-10)

hibidores de proteasas:

Inhibidores de la elastasa de neutrófilos

nhibidores de la catepsina

nhibidores de las metaloproteinasas de matriz

Elafina

Inhibidor de la leucoproteasa secretora 
do el posible papel de los antagonistas de la IL8, como son los antagonistas específicos de CXCR2, uno de los receptores en los neutrófilos que son activados por la IL8. Otro de los mediadores implicados en la EPOC es el factor de necrosis tumoral $\alpha(\mathrm{TNF} \alpha)$, cuya acción se intenta antagonizar por varios métodos, como son el desarrollo de anticuerpos monoclonales, receptores solubles de TNF que "secuestran" el TNF liberado o bien con inhibidores de la convertasa de TNF.

El estrés oxidativo está aumentado en pacientes con EPOC, particularmente durante las exacerbaciones (57). Hay oxidantes en el humo de los cigarrillos y otros que se producen endógenamente por las células inflamatorias activadas, como macrófagos y neutrófilos. Esto sugiere que los antioxidantes podrían jugar un papel en el tratamiento de la EPOC. $\mathrm{Se}$ ha visto en estudios clínicos que la $\mathrm{N}$-acetilcisteína, la cual tiene acción antioxidante in vitro e in vivo, reduce el número de exacerbaciones de la EPOC y en un estudio no controlado parecía reducir la velocidad de descenso del $\mathrm{FEV}_{1}$ en un período de 2 años. Por otro lado, el estrés oxidativo puede dar lugar a la formación aumentada de isoprostanos, los cuales actuando a través de receptores de tromboxanos dan lugar a una potente broncoconstricción in vitro. Por este motivo se ha sugerido que los antagonistas de los receptores de tromboxanos pueden ser también beneficiosos en la EPOC.

2. Nuevos tratamientos antiinflamatorios. Como ya hemos visto previamente en esta revisión, los corticoides inhalados no han demostrado un beneficio significativo en ralentizar la progresión de la EPOC. Esto puede ser debido a que son inefectivos en la inflamación neutrofílica y a que prolongan la supervivencia de los neutrófilos al inhibir la apoptosis. Hay actualmente otros tratamientos antiinflamatorios en estudio, entre los que destacan los inhibidores de la fosfodiesterasa 4 (PDE-4 ); éstos tienen un efecto inhibitorio en células inflamatorias claves implicadas en la EPOC, como son los neutrófilos, macrófagos y linfocitos $\mathrm{T}$ citotóxicos. Una limitación importante de estos fármacos, sobre todo en los de $1^{\mathrm{a}}$ generación, es la aparición frecuente de náuseas. Un inhibidor de PDE-4 de $2^{\text {a }}$ generación (SB 207499) ha demostrado una mejoría en los síntomas y la función pulmonar en pacientes con EPOC moderada.

Otros tratamientos antiinflamatorios nuevos, que actúan sobre la inflamación neutrofílica y que están en estudio, aunque sin haber alcanzado ensayos clínicos incluyen: inhibidores de factor nuclear $\kappa \beta$ (NF- $\kappa \beta)$, inhibidores de las moléculas de adhesión e interleucina 10 (IL-10), entre otros.

3. Inhibidores de proteasas. En la EPOC existe un disbalance entre las proteasas que digieren la elastina y otras proteínas estructurales, y las antiproteasas que contrarrestan esta acción. Esto sugiere que inhibir los enzimas proteolíticos o bien aumentar las antiproteasas puede ser beneficioso y teóricamente debería prevenir la progresión de la EPOC.

La elastasa de los neutrófilos participa de forma muy activa en la actividad elastolítica pulmonar, estimula además la secreción de moco y la liberación de IL-8 y puede perpetuar el estado inflamatorio. Hay varios inhibidores de la elastasa de los neutrófilos en desarrollo, aunque sólo uno ha sido probado clínicamente. El MR889 administrado por vía oral durante 4 semanas no mostró ningún efecto sobre la presencia de péptidos derivados de elastina en plasma o desmosina urinaria (ambos marcadores de actividad elastolítica), aunque pueden no ser marcadores sensibles (58).

Aunque la elastasa de los neutrófilos es el enzima elastolítico mayor en pacientes con déficit de $\alpha 1$ antitripsina ( $\alpha 1$ AT) puede no serlo en la EPOC relacionada con el hábito tabáquico. Es importante por tanto considerar la inhibición de otros enzimas como la catepsina $\mathrm{G}$ y la proteinasa 3, que también tienen actividad elastolítica. La suramina es un potente inhibidor de catepsina $\mathrm{G}$, proteinasa 3 y elastasa de los neutrófilos. Están ahora en desarrollo inhibidores de catepsina nuevos y más específicos.

Las metaloproteinasas de matriz (MMPs) son un grupo de más de 20 endopeptidasas capaces de degradar todos los componentes de la matriz extracelular del parénquima pulmonar. Los enzimas endógenos responsables de contrarrestar dicha acción son los inhibidores tisulares de metaloproteinasas (TIMPs). En la búsqueda de nuevos tratamientos que modifiquen la historia natural de la EPOC se están intentando las 2 aproximaciones posibles: una, inhibir las MMPs; otra, aumentar la secreción de TIMPs. Se han desarrollado varios inhibidores no selectivos de las metaloproteinasas de matriz y hay una búsqueda para inhibidores que sean más selectivos y que no tengan los importantes efectos secundarios músculo-esqueléticos que acompañaban frecuentemente a los primeros.

Otra aproximación es la suplementación con antiproteasas endógenas, tales como la $\alpha 1$ AT, el inhibidor de la leucoproteasa secretora o la elafina, bien mediante la administración de productos recombinantes humanos o incluso por terapia génica.

Aparte de la búsqueda de nuevos fármacos, es probable que se necesiten nuevos sistemas para la administración de los mismos. Los dispositivos de inhalación actuales han sido diseñados para liberar los medicamentos a las vías aéreas de conducción en pacientes con asma. Sin embargo, en la EPOC el proceso inflamatorio se localiza sobre todo en las vías aéreas periféricas y el parénquima pulmonar, los cuales puede que no se alcancen de forma óptima con los dispositivos actuales. Es posible que fármacos de administración sistémica o nuevos dispositivos de inhalación que liberen partículas aerosolizadas más pequeñas sean más útiles en la EPOC.

\section{Bibliografía}

1. Barnes PJ. Medical Progress: Chronic obstructive pulmonary disease. $\mathrm{N}$ Engl J Med 2000; 343: 269-80.

2. Ferguson GT, Cherniack RM. Current concepts: Management of COPD. N Engl J Med 1993; 328: 1017-22.

3. Pauwels RA, Buist AS, Calverley PMA, Jenkins CR, Hurd SS. Global strategy for the diagnosis, management, and prevention of chronic obstructive pulmonary disease: NHLBI/WHO Global Initiative for Chronic

Obstructive Lung Disease (GOLD) workshop summary. Am J Respir Crit Care Med 2001; 163: 1256-76.

4. Madison Jm, Irwin RS. Chronic obstructive pulmonary disease. Lancet 1998; 352: 467-73

5. American Thoracic Society. Standards for the diagnosis and care of patients with chronic obstructive pulmonary disease. Am J Respir Crit Care Med 1995; 152: S77-121. 
6. Celli BR. Criterios actuales para el tratamiento de la enfermedad pulmonar obstructiva crónica. En: Dosman JA, Cockcroft DW, eds. Clínicas Médicas de Norteamérica. México: McGraw-Hill Interamericana, 1996; 557-76.

7. Anthonisen NR, Connett JE, Kiley JP, et al. Effects of smoking intervention and the use of an inhaled anticholinergic bronchodilator on the rate of decline of $\mathrm{FEV}_{1}$ : the Lung Health Study. JAMA 1994; 272: 1497-505.

8. Silagy C, Mant D, Fowler G, Lodge M. Meta-analysis on efficacy of nicotine replacement therapies in smoking cessation. Lancet 1994; 343: 139-42.

9. Jorenby DE, Leischow SJ, Nides MA, et al. A controlled trial of sustained-release bupropion, a nicotine patch, or both for smoking cessation. $\mathrm{N}$ Engl J Med 1999; 340: 685-91.

10. O’Donnell DE, Lam M, Webb KA. Measurement of symptoms, lung hyperinflation, and endurance during exercise in chronic obstructive pulmonary disease. Am J Respir Crit Care Med 1998; 158: 1557-65.

11. Ferguson GT. Update on pharmacologic therapy for chronic obstructive pulmonary disease. En: Rochester CL, ed. Clinics in Chest Medicine. Philadelphia: WB Saunders, 2000; 723-38.

12. Rennard SI, Serby CW, Ghafouri M, Johnson PA, Friedman M. Extended therapy with ipratropium is associated with improved lung function in patients with COPD. A retrospective analysis of data from seven clinical trials. Chest 1996; 110: 62-70.

13. Deffebach ME. Bronchodilator therapy of COPD. A reanalysis suggests new roles and new concerns. Chest 1996; 110: 5-6.

14. COMBIVENT Inhalation Aerosol Study Group. In chronic obstructive pulmonary disease, a combination of ipratropium and albuterol is more effective than either agent alone. An 85-day multicenter trial. Chest 1994; 105: 1411-9.

15. Rennard SI, Anderson W, Zu Wallack R, et al. Use of a long-acting inhaled beta2-adrenergic agonist, salmeterol xinafoate, in patients with chronic obstructive pulmonary disease. Am J Respir Crit Care Med 2001; 163: 1087-92.

16. Mahler DA, Donohue JF, Barbee RA, et al. Efficacy of salmeterol xinafoate in the treatment of COPD. Chest 1999; 115: 957-65.

17. Maesen BLP, Westermann CJJ, Duurkens VAM, van den Bosch JMM. Effects of formoterol in apparently poorly reversible chronic obstructive pulmonary disease. Eur Respir J 1999; 13: 1103-8.

18. Dowling RB, Johnson M, Cole PJ, Wilson R. Effect of salmeterol on Haemophilus influenzae infection of respiratory mucosa in vitro. Eur Respir J 1998; 11: 86-90.

19. Barnes PJ. Beta-adrenergic receptors and their regulation. Am J Respir Crit Care Med 1995; 152: 838-60.

20. Torphy TJ. Phosphodiesterase isozymes: Molecular targets for novel antiasthma agents. Am J Respir Crit Care Med 1998; 157: 351-70.

21. Shannon M. Life-threatening events after theophyline overdose: A 10year prospective analysis. Arch Intern Med 1999; 159: 989-94.

22. Pauwels RA, Löfdahl C-G, Laitinen LA, et al. Long-term treatment with inhaled budesonide in persons with chronic obstructive pulmonary disease who continue smoking. N Engl J Med 1999; 340: 1948-53.

23. Vestbo J, Sorensen T, Lange P, Brix A, Torre P, Viskum K. Long-term effect of inhaled budesonide in mild and moderate chronic obstructive pulmonary disease: a randomised controlled trial. Lancet 1999; 353: 1819-23.

24. Burge PS, Calverley PM, Jones PW, Spencer S, Anderson JA, Maslen TK. Randomised, double blind, placebo controlled study of fluticasone propionate in patients with moderate to severe chronic obstructive pulmonary disease: the ISOLDE trial. BMJ 2000; 320: 1297-303.

25. The Lung Health Study Research Group. Effect of inhaled triamcinolone on the decline in pulmonary function in chronic obstructive pulmonary disease. N Engl J Med 2000; 343: 1902-9.

26. Mapp CE. Inhaled glucocorticoids in chronic obstructive pulmonary disease. N Engl J Med 2000; 343: 1960-1.

27. Medical Research Council Working Party: Long term domiciliary oxygen therapy in chronic hypoxic cor pulmonale complicating chronic bronchitis and emphysema: report of the Medical Research Council Working Party. Lancet 1981; 1: 681-6.

28. Nocturnal Oxygen Therapy Trial Group. Continuous or nocturnal oxygen therapy in hypoxemic chronic obstructive lung disease: a clinical trial. Ann Intern Med 1980; 93: 391-8.

29. Jurado Gámez B, Muñoz Triano E, Daviña Romero S, del Pino Gómez R, Ortiz Jiménez A, Sans Sánchez M. Oxigenoterapia domiciliaria. Estudio de utilización en un área rural. An Med Interna (Madrid) 1998; 15: 358-62.

30. Chaouat A, Weitzenblum E, Kessler R, et al. A randomised trial of nocturnal oxygen therapy in chronic obstructive pulmonary disease patients. Eur Respir J 1999; 14: 1002-8.

31. Honig EG, Ingram RH Jr. Chronic bronchitis, emphysema, an airway obstruction. En: Fauci AS, Braunwald E, Isselbacher KJ, Wilson JD,
Martin JB, Kasper DL, Hauser SL, Longo DL, eds. Harrison's. Principles of Internal Medicine, 14 ed. New York: McGraw-Hill,1998: 1451-9.

32. Golpe Gómez R, Mateos Colino A, Soto Franco I. Técnica inadecuada en el empleo de inhaladores en pacientes atendidos en una consulta de neumología. An Med Interna (Madrid) 2001; 18: 69-73.

33. Hanania NA, Wittman R, Kesten S, Chapman KR. Medical personnel's knowledge of and ability to use inhaling devices. Chest 1994; 105: 111-6.

34. Bishop MJ. Beta agonist delivery and the outpatient. Chest 1989; 96: 1219-20.

35. Newman SP. Aerosol deposition considerations in inhalation therapy. Chest 1985; 88 (Supl. 2): 152-60.

36. Newman SP, Millar AB, Lennard-Jones TR, Moren F, Clarke SW Improvement of pressurised aerosol deposition with nebuhaler spacer device. Thorax 1984; 39: 935-41.

37. Serra Batlles J, Comella A, Casas JC, Plaza V, Brugués J. Cámaras de inhalación: ¿alternativa al inhalador convencional? Med Clin (Barc) 1993; 101: 125-7.

38. König P. Spacer devices used with metered-dose inhalers. Breakthrough or gimmick? Chest 1985; 88: 276-84

39. Newhouse MT, Nantel NP, Chambers CB, Pratt B, Parry-Billings M Clickhaler (a novel dry powder inhaler) provides similar bronchodilation to pressurized metered-dose inhaler, even at low flow rates. Chest 1999; 115: 952-6.

40. Newman SP, Moren F, Trofast E, Talaee N, Clarke SW. Deposition and clinical efficacy of terbutaline sulphate from Turbuhaler, a new multidose powder inhaler. Eur Respir J 1989; 2: 247-52.

41. Berry RB, Shinto RA, Wong FH, Despars JA, Light RW. Nebulizer vs spacer for bronchodilator delivery in patients hospitalized for acute exacerbations of COPD. Chest 1989; 96: 1241-6.

42. Belman MJ, Kendregan BA. Exercise training fails to increase skeletal muscle enzymes in patients with chronic obstructive pulmonary disease. Am Rev Respir Dis 1981; 123: 256-61.

43. Casaburi R, Patessio A, Ioli F, Zanaboni S, Donner CF, Wasserman K. Reductions in exercise lactic acidosis and ventilation as a result of exercise training in patients with obstructive lung disease. Am Rev Respir Dis 1991; 143: 9-18.

44. Wingate BJ, Hansen-Flaschen J. Anxiety and depression in advanced lung disease. Clinic Chest Med 1997; 18: 495-505.

45. Smoller JW, Pollack MH, Otto MW, Rosenbaum JF, Kradin RL. Panic anxiety, dyspnea, and respiratory disease: Theoretical and clinical considerations. Am J Respir Crit Care Med 1996; 154: 6-17.

46. Douglas NJ. Sleep in patients with chronic obstructive pulmonary disease. Clin Chest Med 1998; 19: 115-25.

47. Prevention and control of influenza: recommendations of the Advisory Committee on Immunization Practices (ACIP). MMWR Morb Mortal Wkly Rep 2000; 49 (RR-3): 1-38.

48. Prevention of pneumococcal disease: recommendations of the Advisory Committee on Immunization Practices (ACIP). MMWR Morb Mortal Wkly Rep 1997; 46 (RR-8): 1-24.

49. Nichol KL, Baken L, Nelson A. Relation between influenza vaccination and outpatient visits, hospitalization, and mortality in elderly persons with chronic lung disease. Ann Intern Med 1999; 130: 397-403.

50. Wright P. Influenza in the family. N Engl J Med 2000; 343: 1331-2.

51. Winquist AG, Fukuda K, Bridges CB, Cox NJ. Neuraminidase inhibitors for treatment of influenza A and B infections. MMWR Morb Mortal Wkly Rep 1999; 48 (RR-14): 1-9.

52. Flaherty KR, Martínez FJ. Lung volume reduction surgery for emphysema. En: Rochester CL, ed. Clinics in Chest Medicine. Philadelphia: WB Saunders 2000: 819-48.

53. Geddes D, Davies M, Koyama H, et al. Effect of lung-volume-reduction surgery in patients with severe emphysema. N Engl J Med 2000; 343: 239-45.

54. National Emphysema Treatment Trial Research Group. Patients at high risk of death after lung-volume-reduction surgery. N Engl J Med 2001; 345: 1075-83.

55. Barnes PJ. Novel approaches and targets for treatment of chronic obstructive pulmonary disease. Am J Respir Crit Care Med 1999; 160: S72-79.

56. Díez Herranz A. Manejo de la enfermedad pulmonar obstructiva crónica. ¿Hay realmente más consenso, o tan sólo hay más consensos? An Med Interna (Madrid) 1998; 15: 44-6.

57. Repine JE, Bast A, Lankhorst I. Oxidative stress in chronic obstructive pulmonary disease. Am J Respir Crit Care Med 1997; 156: 341-57.

58. Luisetti M, Sturanic C, Sella D, et al. MR 889, a neutrophil elastase inhibitor in patients with chronic obstructive pulmonary disease: a double blind randomized, placebo-controlled clinical trial. Eur Respir J 1996; 9: 1482-6. 